\title{
Structural and Functional Consequences of Three Cancer- Associated Mutations in the Oncogenic Phosphatase Shp2
}

\author{
Jonathan R. LaRochelle, ${ }^{\dagger \ddagger}$ Michelle Fodor, ${ }^{\#}$ Xiang Xu, ${ }^{\dagger \ddagger}$ Izabela Durzynska, ${ }^{\dagger \ddagger}$ Lixin Fan, ${ }^{\Uparrow}$ Travis \\ Stams, ${ }^{\#}$ Ho Man Chan, ${ }^{* *}$ Matthew J. LaMarche, ${ }^{*}$ Raj Chopra, ${ }^{*}$ Ping Wang, ${ }^{* *}$ Pascal D. Fortin, ${ }^{* *}$ \\ Michael G. Acker, ${ }^{* *}$ and Stephen C. Blacklow ${ }^{\dagger \ddagger}$ \\ ${ }^{\dagger}$ Department of Biological Chemistry \& Molecular Pharmacology, Harvard Medical School, Boston, MA 02115, USA \\ ${ }^{\ddagger}$ Department of Cancer Biology, Dana-Farber Cancer Institute, Boston, MA 02215, USA \\ ${ }^{*}$ Center for Proteomic Chemistry, ${ }^{* *}$ Oncology, ${ }^{*}$ Global Discovery Chemistry, Novartis Institutes for Biomedical Research, \\ Cambridge, MA, 02139,USA \\ " Basic Science Program, Leidos Biomedical Research, Inc., Frederick National Laboratory for Cancer Research, Frederick, MD \\ 21702, USA
}

\section{SUPPLEMENTARY INFORMATION}

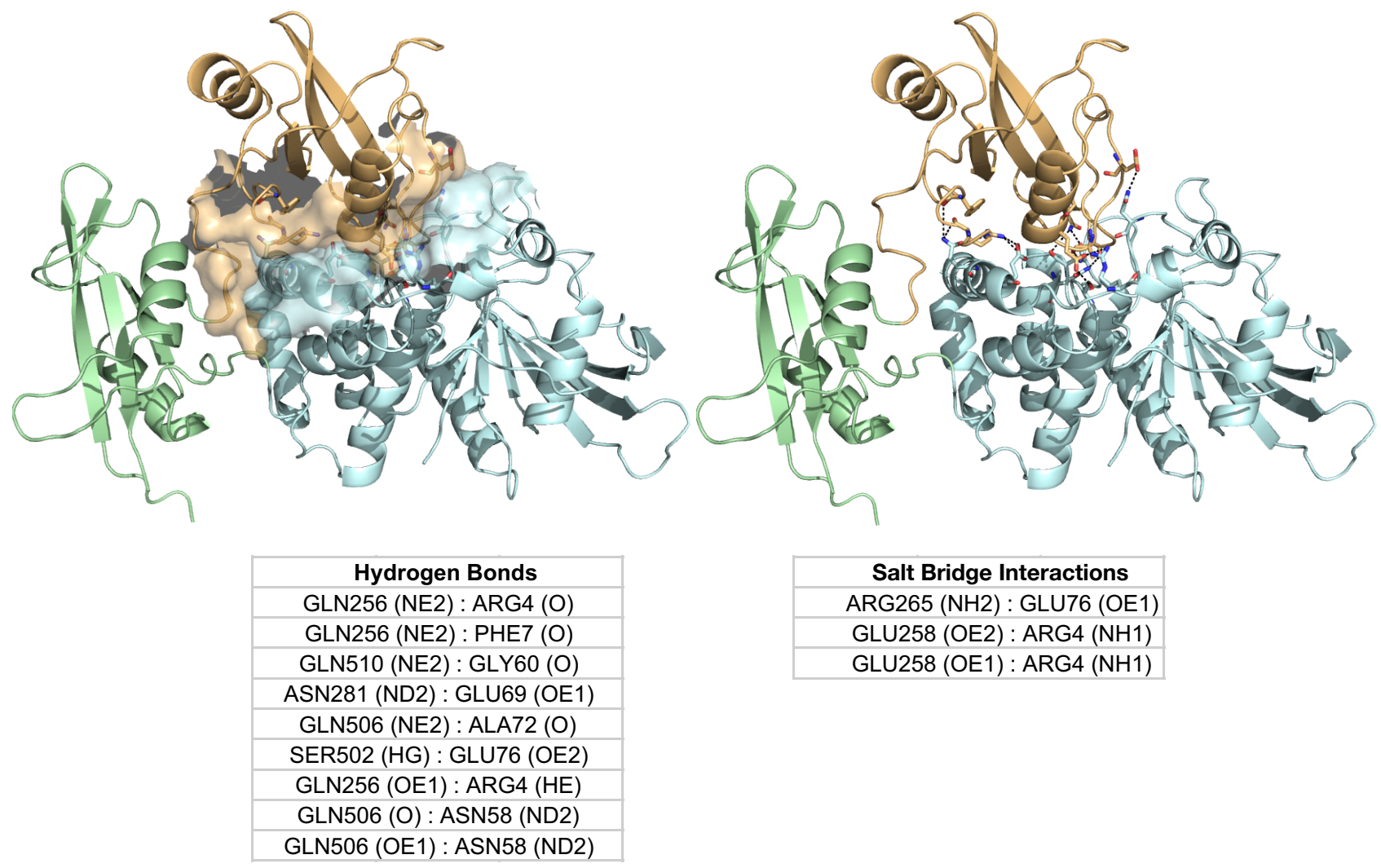

Supplemental Figure 1. The buried surface area between N-SH2 and PTP domains involves 70 residues (32 from N-SH2 and 38 from PTP) and covers $1176 \AA^{2}$. There are 9 hydrogen bond and 3 salt bridge contributions to this interface. PDB: 2SHP and QtPISA $^{1}$ were used for this analysis. 


\section{References}

[1] Krissinel, E., and Henrick, K. (2007) Inference of macromolecular assemblies from crystalline state, J Mol Biol 372, 774-797. 